\title{
The Model-less Neuromimetic Chip and its Normalization of Neuroscience and Artificial Intelligence
}

This paper was downloaded from TechRxiv (https://www.techrxiv.org).

\section{LICENSE}

CC BY-NC-SA 4.0

SUBMISSION DATE / POSTED DATE

29-11-2020 / 04-01-2021

CITATION

Hales, Colin (2020): The Model-less Neuromimetic Chip and its Normalization of Neuroscience and Artificial Intelligence. TechRxiv. Preprint. https://doi.org/10.36227/techrxiv.13298750.v3

$\mathrm{DOI}$

10.36227/techrxiv.13298750.v3 


\title{
The Model-less Neuromimetic Chip and its Normalization of Neuroscience and Artificial Intelligence
}

\author{
Colin G. Hales \\ Department of Anatomy and Neuroscience, University of Melbourne, Victoria, Australia \\ Email: cghales@ unimelb.edu.au
}

\section{Keywords}

Machine intelligence; Artificial Intelligence; Neuroscience; General-purpose Computer; Neuromorphic chip; Neuromimetic chip;

\begin{abstract}
The conceptual basis of a novel neuromimetic chip is described. Based on an existing computational bioelectrodynamics study and adaptive brain signaling biophysics knowledge from neuroscience, the chip is, in effect, a form of inorganic artificial brain tissue. This 'physics replication' approach involves no abstract models of brain tissue physics or function. Instead of the physics of a general-purpose computer or the physics of abstract models of the brain on the chip (analogue or digital), this neuromimetic chip has an inorganic version of natural adaptive brain signaling physics. As a result of using the native brain physics, the chip has functionally relevant endogenous quasistatic electric and magnetic field systems of the form known to be expressed by excitable cell tissue. Fully developed at macroscopic scales it can be expected to produce an EEG/MEG-like electromagnetic signature. This article does an extended analysis to understand an observed generalized lack of the physics-replication approach and its implications for the neuroscience of natural and artificial intelligence. This is achieved through a technical comparison with the neuromimetic chip's closest relative, the neuromorphic chip (of the class of general-purpose computers). The results indicate that the physics-replication approach is a possible but neglected option. It also reveals that the neuromimetic chip contributes empirical science, in contrast to the theoretical science conducted using general-purpose computers. Because of the chip's novelty and proximity to foundational issues, the article contributes necessary background information in anticipation of the arrival of the first prototyping results over the coming years.
\end{abstract}

\section{Introduction}

This article introduces a novel electronic, neuromimetic chip hardware concept. It is a neuroscience/engineering initiative targeting the neuroscience of excitable cells, and thereby the scientific study of natural and artificial intelligence (AI). It differs from existing general-purpose (GP) computer hardware (defined in Supplementary 1-1) in that it is, in effect, inorganic artificial excitable cell tissue. This proposal is more than merely a machine intelligence solution. The chip proposal fundamentally alters neuroscience in a general sense. For the purposes of distinguishing the new chip from existing neuromimetic proposals found in the literature, we are going to call the chip 'Xchip'. The novelty and technological basis of Xchip is to be delivered in a container of an analysis of its role in neuroscience and the science of artificial intelligence (AI). This is aimed at ensuring that the novel position of Xchip within science is properly understood. The Xchip design is categorically distinct, in a provable way, from previous hardware. In fully conveying this uniqueness, prototyping results for Xchip will then be met by a community that has been adequately prepared.

The science of AI is generally agreed to have been born around the time of the famous Dartmouth conference in 1956 (McCarthy et al., 1955;Moor, 2006). At roughly age 65, the still youthful science 
of AI, in being confined to the use of GP-computers since birth, is demonstrated here to have been operating all along as a form of theoretical science. This fact of the science, and its practical impact, will also shortly be demonstrated to be anomalous, unique, and unprecedented in science. Xchip enters the scene as a corrective action that introduces empirical science currently missing from neuroscience. This anomalous neuroscience absence then manifests as a missing kind of 'empirical science of AI' that does not use GP-computers. To formally identify this absence, however, requires a calibrated understanding of the basics of scientific behavior. In scientific behavior, empirical observation and theoretical science face-off normally in the following three familiar science contexts:

(i) Observation of a natural context (empirical science).

(ii) Observation of artificial versions of the natural context. Call this engineered or replicated nature a 'scientifically-artificial' version of nature (empirical science).

(iii) Creation of abstract models predictive of properties of the natural context observable in (i) and (ii) (theoretical science).

Activities (i)-(iii) meet each other in a mutual, reciprocating, iterative distillation that converges on empirically proved 'laws of nature' that are then published in the literature (Rosenblueth and Wiener, 1945;Hales, 2014). A visual depiction of five instances of the science process is shown in Figure 1.

In the central column of Figure 1 is a (i) natural context. In the left column is (ii), a 'scientificallyartificial' version of (i) called a replicant. It is created, through retention of the targeted physics of interest, to replicate some subset (or all) of the physics of (i) so that an aspect of the function of (i) is physically conserved for study/engineering purposes. In the right column is a (iii) 'law of nature', $\mathbf{t}$, an abstract model of the targeted natural regularity in (i) and (ii). For centuries of science, the numeric/symbolic exploration of $\mathbf{t}$ was done by hand. In the 1940s, electronic GP-computers automated the exploration. Either way, in Figure 1 the exploration process is signified as the operation of an emulant (sometimes called a simulation). A detailed description of how the (i)/(ii)/(iii) framework operates, including how it was used to empirically establish the framework itself, is included in Supplementary 2.

The last entry in Figure 1, the (e) brain and the neuroscience of natural intelligence, is our context of interest. Figure 1(e) displays the claimed missing part of the neuroscience as the Xchip-based replicant, the (ii) empirical science of a scientifically-artificial version of the (i) natural brain. The physics basis of (ii) is detailed shortly. If effect, creating (ii) Xchip creates inorganic artificial brain tissue hardware that is not a listed GP-computer (Supplementary 1-1). Instead of the physics of a GP-computer (or an abstract model of (i) in chip form) on its (iii) chip hardware, (ii) neuroscience puts an inorganic version of (i) natural brain signaling physics on the chip hardware. The resultant Xchip can be expected to have an endogenous EEG (electric field, electroencephalogram) and MEG (magnetic field, magnetoencephalogram) signature like us. Because of this and the lack of abstract models, Xchip is formally entitled to the (ii) replicant position as a form of empirical science.

At this point, many readers will have experienced a strong dissonance between a long-accepted notion of an artificial thing (Figure 1(e)(iii) emulant) and a novel and unfamiliar claimed artificial version of a natural thing (Figure 1(e)(ii) replicant). Formally demonstrating this difference and its implications is the main job of this article. The fact is, it is possible to build a Figure 1(e)(ii) artificial version of (i) natural brain tissue and clearly prove a measurable physical difference between it and what is happening under Figure 1(e)(iii). Claims about the conditions of Figure 1(e)(i)/(ii)/(iii) functional equivalence are the ultimate points of unresolved contention in the science. The Figure 1(e)(i) natural brain is the first and possibly only place in nature where equivalence between Figure 1(i) nature and a Figure 1(iii) emulant has been hypothesized by a science community. So far that equivalence is 
unproved and has been assumed while lacking the empirical testing that formally proves it under Figure 1(e)(ii). Exploring the Figure 1(e)(i) $\Leftrightarrow$ (iii) physical/functional equivalence through introduction of the Figure 1(e)(ii) neuromimetic Xchip is the goal of chip prototyping over the coming years. Clarifying the context of the (i) $\Leftrightarrow$ (iii) equivalence is deeply impoverished when Figure 1(e)(ii) is missing from the science. Figure 1(e)(ii) is not a Figure 1(e)(iii) GP-computer and is long overdue for introduction to the science.

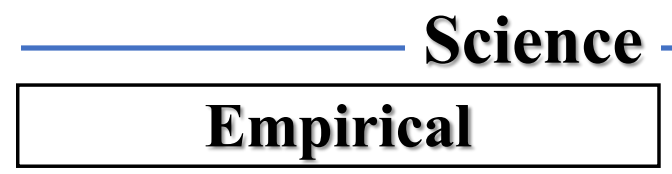

(ii)

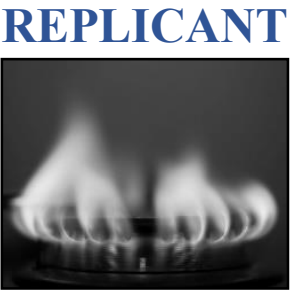

(b)

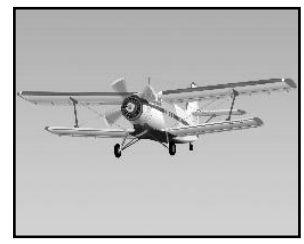

(c)

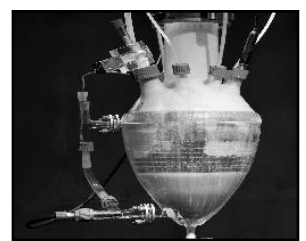

(d)

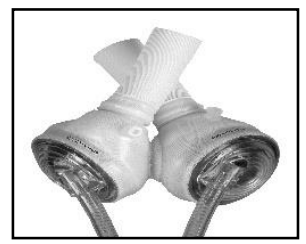

(e)

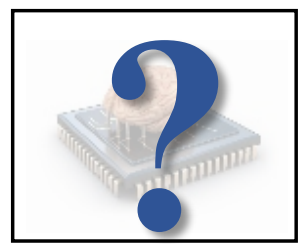

(i)
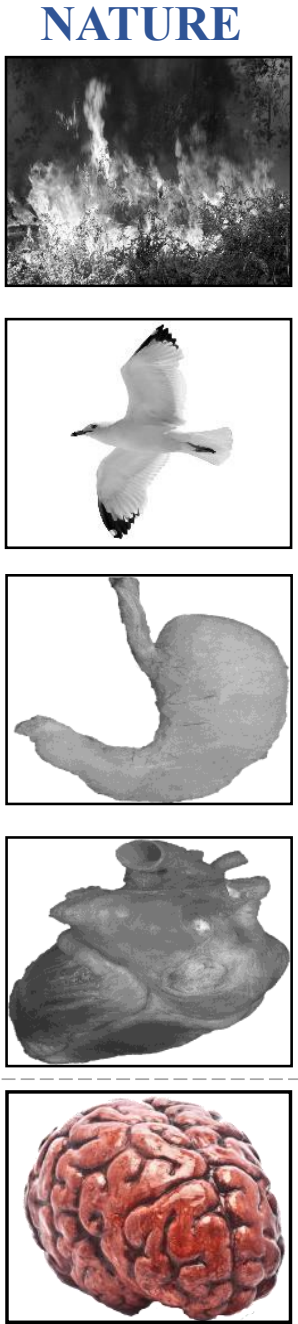

\section{Theoretical}

(iii)
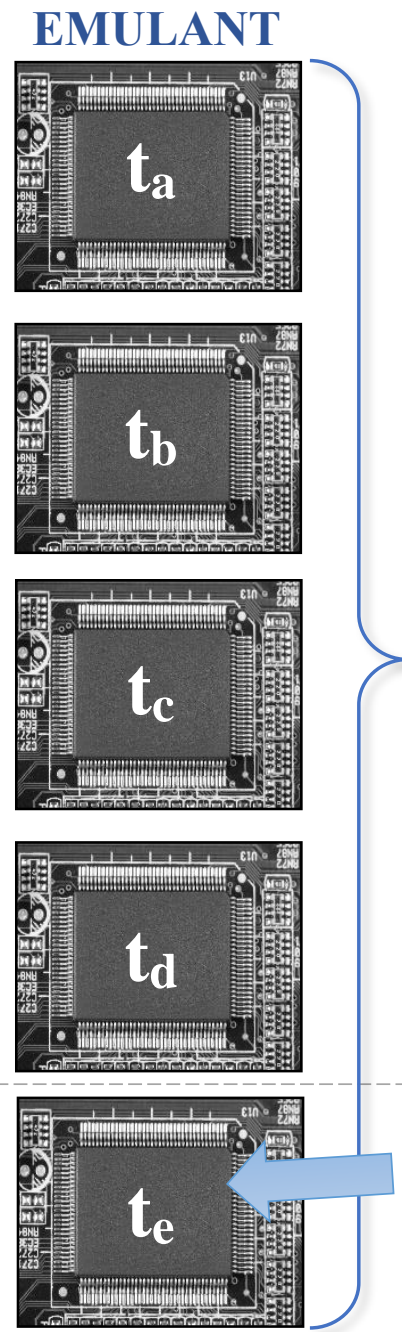

'Artificial intelligence' (AI)

\section{NEUROSCIENCE of NATURAL INTELLIGENCE}

Figure 1. Examples of the (i)-(iii) structure of scientific behavior applied to five natural phenomena. In the middle column is (i) empirical science of natural (a) combustion, (b) flight, (c) human stomach, (d) human heart, (e) human brain. In column (ii) is empirical science of an inorganic scientifically-artificial version of the natural original. Column (iii) depicts theoretical science involving abstract models $\mathbf{t}$ (theories) of (i) and (ii). These are explored using GP-computers. In (e) we see (i) natural excitable cell tissue, (ii), where Xchip (neuroscience-originated AI) would be if it existed and (iii), where current AI is centered. Based on (Hales, 2014, Figure 14.1) and (Rosenblueth and Wiener, 1945). 
With Xchip introduced to Figure 1(e)(ii) neuroscience, a pair of AI anomalies becomes apparent. First, AI could be produced by neuroscience under (ii). This 'neuroscience-originated' AI is currently missing. That is, the Figure 1(e)(ii) neuroscience lack can be seen to have been inherited by the science of AI. An extensive longitudinal search of the scientific literature, back to the inception of AI in the mid-1950s reveals that contributions to Figure 1(e)(ii) do not exist. The requisite (ii) physics replication part of empirical neuroscience does not make an appearance anywhere in the literature. Nor does it make an appearance as a form of potential AI. No instance has been found in searches done to date. An absence of (ii) empirical science is not unprecedented in the general science of natural phenomena. For example, we cannot yet make artificial gravitational waves. In general, there may be many reasons for a lack of (ii) empirical science, technical and otherwise. However, if we can create (ii) and do not do it, usually there are reasons for the lack and an awareness of it is visible in the literature. This is not the case in the (ii) neuroscience of excitable cells. A proof that no instance of 1(e)(ii) exists is provided in the coming sections.

The second anomaly involves the (iii) GP-computer-exploration of abstract models of the Figure 1(e)(i) brain function being called 'artificial intelligence'. Notice that the analogous kind of functional equivalence does not apply to Figure 1(a)(iii)-(d)(iii). None of Figure 1(a)(iii)-(d)(iii) are artificial versions of their (i) natural counterpart. This lack of (i) $\Leftrightarrow$ (iii) equivalence is generalized across all the sciences of natural phenomena. Extensive searching has (so far) failed to find any science where a (iii) GP-computed abstract theoretical model of a natural phenomenon is literally a functional identity with the (i) natural phenomenon. For example, an artificial (i) kidney is a (ii) dialysis machine including actual filtration physics, not a (iii) GP-computed exploration of abstract filtration physics. And so forth. The natural brain is, prima facie, no different. Some as yet unknown aspect of its nonlinear, thermodynamically far-from-equilibrium complexity-physics may be just as essential to brain function as filtration physics is to kidney function. Nobody can yet formally claim to know what the brain's essential physics is and what goes missing if it is not retained. To eliminate the (i) fundamental signaling physics of the brain in the context of the Figure 1(e)(iii) use of GP-computers, is to assume that there is no (i) endogenous brain physics essential to brain function. This kind of 'physicsindependence' (not to be confused with substrate/material independence - see Supplementary 2-2(ii)) is unprecedented in the science of natural phenomena. This formally confirms a potential equivalence between (i) and (iii), in the case of the brain, as unprecedented in science and unique to Figure 1(e) neuroscience.

As anomalous and unique a potential (i) $\Leftrightarrow$ (iii) equivalence may be for the brain, it must still be granted as possible in principle. The brain may be different. We will later describe a detailed technical mechanism for (ii) $\Leftrightarrow$ (iii) confusion unique to the brain that is consistent with (predicts) the presence of the above double anomaly.

Accompanying the anomalous structure of the Figure 1(e) neuroscience, over the same time period is a continuing unresolved and robustly contested controversy about the equivalence of (i) brains and (iii) GP-computers e.g. (McCarthy et al., 1955;Dreyfus, 1972;Searle, 1980;Brooks et al., 2012;Milkowski, 2017;Fjelland, 2020). Xchip is not a 'GP-computer' in the sense of not being in the Supplementary 11 list. But the classification itself is not the issue here. What uniquely distinguishes Xchip is that there is literally and only brain physics involved, where there is none in any of the members of the Supplementary 1-1 GP-computer list. The Xchip concept speaks to the long argument in the literature. It does this by adding Figure 1(e)(ii) so that the potential (i) $\Leftrightarrow$ (iii) equivalence issue can be empirically explored and settled (discovered) because of it. An argument that the brain is/is not some kind of 'GPcomputer' is unlikely to be conclusive until the neuroscience is properly established to empirically 
prove it. In this light, resolution of the long argument in the literature can be seen to be, at least in part, impoverished by the lack of the Xchip option and its (ii) empirical science.

The optimal way to see how the absence of Figure 1(e)(ii) neuroscience has impacted the science of $\mathrm{AI}$ is to describe a fictitious world where neuroscience is normal and consequently the science of AI is normal. This occurs in section 2, which ends by describing the conceptual (physics) basis for the most primitive form of processing element in Xchip. In doing so it reveals the probable technical origins of the anomalous Figure 1(e) (i) $\Leftrightarrow$ (iii) equivalence issue outlined above. A subtle technical confusion is revealed in the specific case of the neuromorphic chip, which is the closest relative to Xchip in the list of GP-computers. Section 3 demonstrates the difference between the neuromorphic chip (GPcomputer/emulant, (iii) theoretical science) and the neuromimetic Xchip (replicant, (ii) empirical science). It does this using the results of an existing computational study of excitable cell electromagnetic (EM) field expression. Once demonstrated in the specific case of the neuromorphic GP-computer, the (ii) $\Leftrightarrow$ (iii) distinction easily generalizes to the rest of the GP-computers listed in Supplementary 1-1. Section 4 then characterizes the operation of the science of AI over the past 65 years. Section 5 details how AI functions before and after being normalized using Xchip. Section 6 signs off by concluding that this article acts as necessary preparation for the various stakeholder communities to encounter the arrival of the first Xchip development results.

\section{Xworld: Has no GP-computers, but still has AI}

For argument purposes, consider a fictitious alternate reality, 'Xworld'. Xworld is similar to our world except that GP-computers do not exist. Computer science as we know it does not exist. Yet, in Xworld, AI is alive and well in 2020 and is instead a product of normal (ii) empirical neuroscience. In Xworld, theoretical science, engineering design, and everything else we use GP-computers for, are carried out by vast armies of the original 'human computers' of the $1940 \mathrm{~s} / 50 \mathrm{~s}$ era depicted in (Turing, 1950; Turing, 1964). The 'human computer' is an extinct profession in our world. My aunt was one of these 'computers'. In 2020 in Xworld this ubiquitous, manual 'human-cloud' computing is universally used. Despite a total lack of Supplementary 1-1 GP-computers, Xworld science continued normally from the 1940s onwards, developing semiconductors and the familiar chip foundries that fabricate them. The only gedanken-experiment weirdness in Xworld is a contrived blind-spot. Somehow Xworld remains oblivious to the potential for GP-computers based on semiconductors or anything else. This strange blind-spot is no less strange than the real Figure 1(e)(ii) blind-spot we have in neuroscience/AI today.

\subsection{Neuroscience in Xworld}

In Xworld, (i) wet neuroscience of excitable cells also proceeds normally. The (iii) theoretical exploration of its abstract models (predictions) of brain tissue properties/function is performed by the human-cloud. Theory (prediction) and nature (measurement) meet in the three usual ways listed in the introduction. However, in Xworld, chip foundries progress differently. Progress is driven by pressure from (ii) neuroscience to put brain signaling biophysics, literally in inorganic form, on the chips. This is used to create inorganic artificial versions of the organic brain tissue found in the wet-lab. The chips produced by Xworld neuroscientists are the previously discussed Xchip. There are no abstract models of the brain involved in Xchip, in either hardware or software form. Using natural nervous tissue as a design template, Xworld engineers use the human-cloud to explore the design of each Xchip and then chip foundries fabricate them.

Xchip is a product of Xworld empirical neuroscience's (ii) exploration of artificial brain tissue to see if properties of the artificial version match the properties found in natural tissue and vice versa. In this 
manner, the specific signaling physics essential to brain function can be delineated, explored and proved in the normal manner of centuries of reductionist science. Xworld neuroscientists view natural brains as a form of natural computation based on the complex dynamics of excitable cell signaling electromagnetism (EM) detailed later. Xchip brains use an inorganic form of exactly the same natural kind of EM, configured in the same way and thereby performing the same 'computations' as natural tissue. Xchip uses electrons as charge carriers whereas the brain uses ions. The resultant EM field systems impressed on space, however, are identical. Specification of the natural brain signaling physics replicated in Xchip will occur shortly. All we say at this point is that whatever it is, it is absent from the various kinds of GP-computers listed in Supplementary 1-1.

Xworld has normal neuroscience that is nevertheless partly unfamiliar to us in our world. Xworld has empirical science in the form of the study of both (i) the natural phenomenon (brain tissue, natural intelligence) and (ii) scientifically-artificial versions of it (Xchip). Just like our science, Xworld also has (iii) theoretical science exploration of abstract models. Unlike our world, however, this is done by the human-cloud. Neuroscience in Xworld is operating normally. Neuroscience in our world is not. This is because in our world, (ii) is missing.

\subsection{AI in Xworld}

Xchip is a form of inorganic artificial brain tissue created by the normal (ii) neuroscience in Xworld. As a result, Xworld researchers realize they incidentally create, in their (ii) empirical work, the possibility of AI as a scientifically-artificial version of natural intelligence based on Xchip. A robot body (a peripheral nervous system) with an Xchip brain (a central nervous system) could become the basis for machine intelligence of all kinds. They could be intelligent and adaptive (up to human level and even beyond), or more mundane, constrained, simple automation. Xworld in 2020, a world in which neuroscience is running normally, has robots with Xchip brains produced by a science of AI originating within neuroscience. This is the neuroscience-originated AI of the introduction. The idea that (iii) theoretical science and the use of 'human-cloud' computed models might somehow be 'artificial intelligence' is something that never occurs to Xworld researchers, and the lack of it doesn't matter to them. Xworld has its smart machines as AI, and GP-computers are not directly involved in the functioning of the machines.

\subsection{Understanding the neuromimetic Xchip and its empirical science}

Up to this point no Xchip design details have been supplied. It is when design details become apparent that we can fully appreciate its distinctness, the subtleties of its position in science and its implications. For brevity, deeper technical detail (including the bulk of this article's references) has been separated into Supplementary 1 (see sections 1-4 and 1-5). We now turn to the central matter of the functional equivalence of (i) brain, (ii) Xchip and the (iii) GP-computer. The (iii) neuromorphic chip (analogue GP-computer, see Supplementary 1-1) provides an ideal contrast-point with (ii) Xchip. For purposes here we will focus on that specific case and assume their equivalence with the (i) brain is a separate matter. Once the (ii) $\Leftrightarrow$ (iii) contrast is demonstrated for the neuromorphic GP-computer, the argument generalizes easily to the rest of the GP-computers in Supplementary 1-1.

Supplementary 1-4 shows how the (ii) Xchip, in comparison to the (iii) neuromorphic chip, reduces to the difference between their fundamental on-chip 'processing elements'. In (ii) the fundamental Xchip processing element replicates the signaling physics (EM) of a patch of active neuron cell membrane. In (iii) the fundamental neuromorphic processing element replicates the physics of a 'lumped-element' electrical equivalent circuit model that predicts a property (the transmembrane voltage) of the exact same (ii) patch of neuron cell membrane. The former is (ii) empirical science (Xchip). The latter is (iii) 
theoretical science in the form of an analogue GP-computer (neuromorphic) that solves for transmembrane voltage and 'writes the result out in voltage-ink'.

In physics terms, the difference is that the EM field systems of these two processing elements are totally unlike each other. Only the elements in (ii) Xchip faithfully replicate the (i) brain's signaling because it replicates its EM field physics basis. It does this using electrically and geometrically accurate replication of the charge density and current density source systems expressed by cell membrane when penetrated by the appropriate kinds of active ion channels. The details of the particular differences in EM field expression originate in a relatively simple difference in the physics of a key component in its account of the cell membrane EM (see Supplementary 1-4 and 1-5). The capacitor in the neuromorphic chip is different to that of Xchip. In Xchip the equivalent to the transmembrane current flows through the dielectric of the capacitor as it does in real cells. In the neuromorphic chip it does not. As a result of engineering this small physical difference, the capacitor in Xchip produces the same EM field system produced by natural cell membrane (both the electric field and magnetic field components). Consequently, any individual processing element (cell membrane patch) within Xchip can interact naturally with its neighbors in 3D, potentially revealing unexpected dynamics not yet included in any (iii) model of it, but that may actually happen in (i) real tissue. That potentiality makes it a form of (ii) empirical science. There are no models or software in Xchip. By contrast, the individual processing elements of the neuromorphic chip have no specific EM field expression. They interact with each other in ways either chosen (wired) by a user of the chip, or in ways adaptively based on automated interconnection rules imposed by a user. Either way, it makes the neuromorphic chip a (iii) exploration of an abstract model domain in contrast to Xchip's exploration of (ii) natural physics. The former is a (iii) emulant. The latter is a (ii) replicant.

It is a matter of an accident of fate that these two physical situations (basic processing element physics), created in hardware, can literally produce identical measurable voltages that in principle also match measurable voltages in the brain. The voltages in Xchip are an emergent property of the replication of the natural EM field system produced by cell membrane. The voltages in a neuromorphic chip are a property of a model (the electrical equivalent circuit model) produced through the use of a totally different and unspecified EM field system (that of the lumped-element electrical components). Supplementary 1-4 and 1-5 detail how this generation of voltages by an neuromorphic analogue computer can be mistaken for physics replication by a naïve observer. Fundamental physics (Maxwell's equations) says that an infinity of different EM field systems can generate the same voltage at a point in space (Hales and Pockett, 2014). Merely replicating voltage does not replicate or model the EM fields that produced it. If that confusion happens, in-principle functional equivalence of the (iii) neuromorphic computer with the (i) brain can be mistakenly inferred as achieved or possible. The radical difference in EM field expression is a subtlety that an experienced electrical engineer, with the appropriate cellular signaling bioelectromagnetism knowledge, circuit theory knowledge and electrical component/chip fabrication knowledge, can find obvious. Others may be less able to see the distinction, which is not speaking to an argument about whether the brain is or can be (is equivalent to) a GPcomputer. It is actually a physics argument speaking to the correct operation of empirical neuroscience.

Note that the replication of the natural atomic-level EM field system, in the first four examples in Figure 1, is also the major underlying reason for the success of all (ii) artificial versions of the (i) nature in the center column. In Xchip we are simply extending the process to the Figure 1(e) brain. 


\section{Computational study: Understanding the endogenous EM expression of neurons}

To demonstrate the difference in EM field expression between the neuromorphic chip (GPcomputer/emulant, (iii) theoretical science) and the neuromimetic Xchip (replicant, (ii) empirical science), we can use the results of an existing computational study of excitable cell EM field expression (Hales, 2011). The study results are examined in three parts:

1. To illustrate the neuromorphic chip, the first part of the study produced the standard view of somatic action potential (SAP) propagation in terms of transmembrane voltage in a (softwareimplemented) compartmental electrical equivalent circuit model (see Supplementary 1-5) of a realistic neuron. It lacks all EM field expression and instead serves to compute transmembrane voltage and currents for all the model compartments. This acquired the data needed for use in the next part of the study.

2. To illustrate Xchip, this study used the compartment currents from the first part. It uses these to examine the subset of the electric field system impressed on space by transmembrane currents during SAP propagation. SAP propagation serves to locally and temporarily disrupt the huge static transmembrane electric field across the lipid bilayer cell membrane of the neuron, thereby producing a specific dynamic, local, faint but spatially large EM field system. The electric field is examined in detail.

3. To further illustrate Xchip, a secondary study was performed on dendritic action potentials (DAP) in the sense of the electric field system impressed on space by a single synapse (a small plaque of ion channels in the post-synaptic cell cleft membrane). When integrated in their thousands with the above SAP field, we get a more accurate perspective on the dynamic portion of the EM field system of a realistic cell with a large population of synapses.

The original study, (Hales, 2011), provided a novel formula for both the electric field and the magnetic field system produced by filamentary current (ion channel) sources embedded within an infinite volume conductor. It is the electric field system that is explored in the original study and presented in studies 2 and 3 above. In practice, Xchip hardware replicates the current sources and in doing so expresses both the electric and magnetic field systems produced by the same sources. Study 2 and 3 illustrate the internal electric field system of a notional Xchip. These two studies can then be compared with the study 1 , which is the product of the operation of a neuromorphic chip, inside which the electric and magnetic fields are irrelevant. This comparison/contrast will happen after the presentation of the study results.

\subsection{Study 1. Neuromorphic chip SAP voltage/current propagation}

The first part of the computational study uses traditional compartmental electrical equivalent-circuit modelling of a single neuron to acquire transmembrane voltages and currents during the propagation of a single somatic action potential throughout the cell. The study used the NEURON simulation package (Hines and Carnevale, 1997;2001;2003) ${ }^{1}$ along with a library neuron package D151 ('EAPS' ModelDB 84589) ${ }^{2}$ that contains a single realistic rat hippocampal CA1 pyramidal neuron. The D151 neuron is delivered decomposed into roughly 1200 compartments. Each compartment was individually populated by the model builders with ion channel currents based on 12 different ion channel types conducting 5 different ion species. The EAPS package was modified to decompose and report, for every time step and every compartment, individual total ion-species currents. The cell was then

${ }^{1}$ https://neuron.yale.edu/neuron/

${ }^{2}$ ModelDB 'EAPS' package.

https://senselab.med.yale.edu/modeldb/getModelFileHtml.cshtml?model=84589\&file=/eaps/docs/index.html 
numerically stimulated to fire a single action potential $10 \mathrm{~ms}$ after the simulation started. Also reported was the dimensions and position of each notional conical frustum compartment. Having collected this data, involvement of the NEURON compartmental simulation software ended.

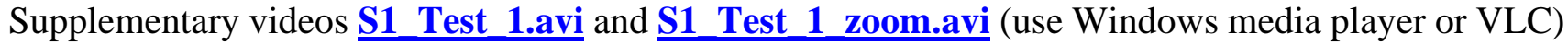
visualize the compartmental transmembrane potential throughout the neuron during the single SAP. If the membrane voltage of the initial segment (one of the soma compartments) is plotted out, we would see the classic transmembrane waveform of a typical action potential (see the host study). The second video shows the first video while zooming in closely on the soma region of the neuron. The static image in Figure 2 shows the neuron potential at the $11.997 \mathrm{~ms}$ mark. Red(+) and blue(-) color illustrates the polarity of each compartment. The resting membrane potential is roughly $-65 \mathrm{mV}$ (blue). The cell video does not last long enough to show the cell's full return to its resting state. Color intensity roughly indicates the magnitude of the transmembrane potential. Notice that not only does the cell potential waveform propagate down the axon (dromic saltatory propagation), it also propagates simultaneously out into the basal dendrites, along the trunk and up to the apical dendrites. The most important feature of Figure 2 is that the electric and magnetic field system play no part in the simulation. This kind of traditional modelling reveals nothing of the EM field impressed on space by the modelled neuron. Nor does it reveal the local field potential (LFP) at points in space outside the neuron. For further detail see (Hales, 2011).

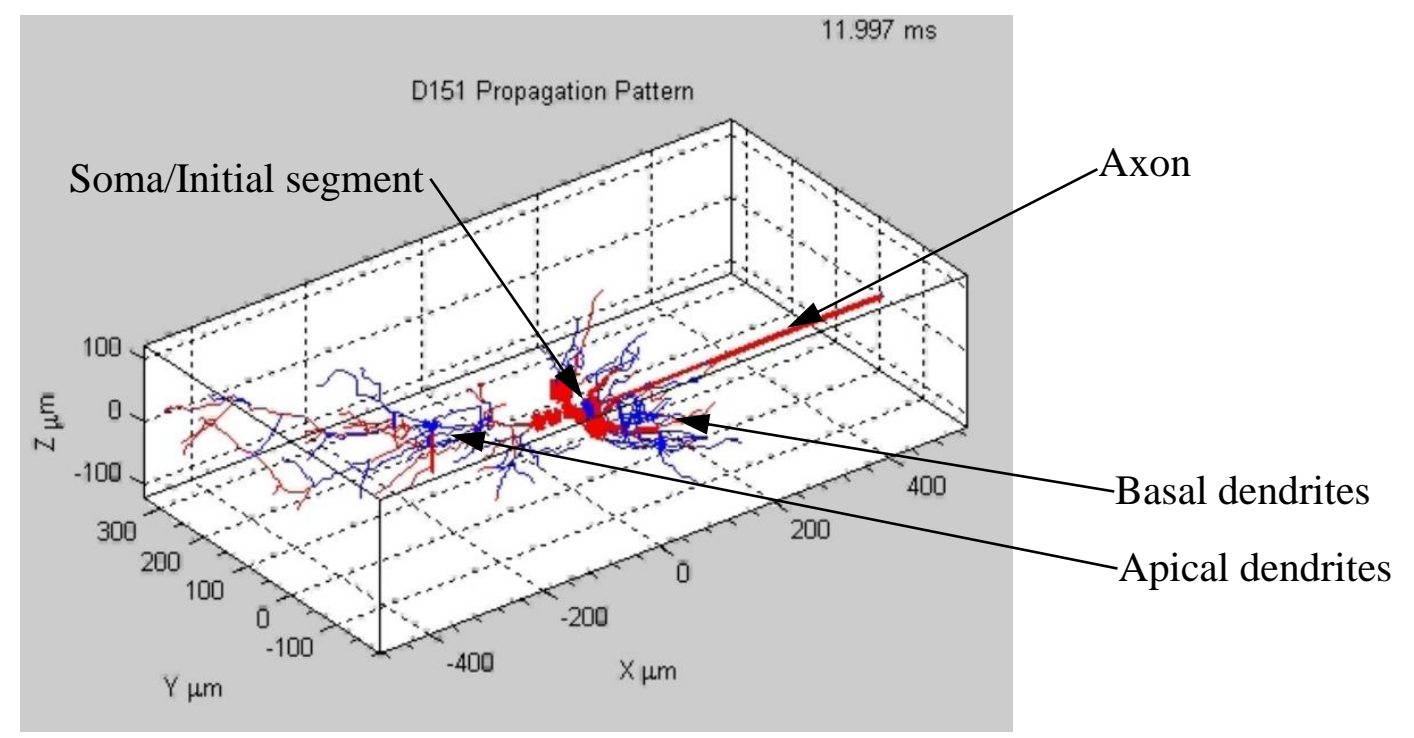

Figure 2. Compartmental equivalent circuit numerical solution produced by the NEURON 'EAPS' simulation. Neuron D151 is decomposed into 1200 compartments (conical frusta). The centerline of each frustum is shown in black. Superimposed compartment color depicts cell voltage polarity $\left(\mathrm{red}^{+} / \mathrm{blue}^{-}\right)$. Red/blue color intensity roughly indicates the magnitude of the transmembrane potential. The neuron was numerically stimulated after letting the model stabilize for $10 \mathrm{~ms}$. The image shows the compartment voltages at the $11.997 \mathrm{~ms}$ mark.

If this neuron D151 was implemented on a neuromorphic chip, then the neuromorphic chip could, inprinciple, produce the same set of compartmental transmembrane potentials. It would do this through implementing the same transmembrane currents. However, the currents are flowing in on-chip electronic devices with EM field expression physics that bears no relation to that of transmembrane ion channel currents. The cell 3D spatial geometry and morphology plays no part in the neuromorphic chip emulant. Compartment connectivity is the only thing conserved by the neuromorphic chip. The solution to the equations for each compartment is represented by voltages inside an analogue computer. 
The compartment interconnections would be mapped into the planar form of the underlying chip compartment hardware array. The function of the neuromorphic chip is that of a general-purpose computer performing the job of solving differential equations for each compartment, albeit with specialized (parallelized) hardware. Whether performed in dedicated neuromorphic hardware or in software (the NEURON package), the results are, in principle, the same. Neither solution involves the EM field expression of neurons or a numerical abstract model of the EM field expression by neurons. Put another way, the production of voltages in the neuromorphic computer is a symbolic representation of voltages that may be found in tissue. The production of voltages inside the neuromorphic chip does not mean that the tissue EM field system, that expresses those same voltages, has been replicated. What we see in Figure 2 is therefore an emulant involved in (iii) theoretical science.

\subsection{Study 2. The electric field produced by a somatic action potential}

The second, novel part of the (Hales, 2011) computational study uses the data from Study 1 (compartmental transmembrane currents and geometry). It took the total transmembrane current for each compartment and divided it equally throughout a set of abstract 'ion channels' in the form of multiple $7.5 \mathrm{~nm}$ current filaments placed around the circular surface of each abstract compartment, orthogonal to the surface. The number of current filaments and their position on the compartment surface was randomized. There were roughly 26000 such 'transmembrane ion channels' spread over the entire outer, circular, abstract 3D surface of all neuron (conical frustum) compartments. The scalar potential field (a property of the vector electric field) for each ion channel was computed (using high performance computing and novel formulae derived from Maxwell's equations under the assumption of volume conduction conditions). Then, all 26000 individual field systems were superposed. This process reveals the general kind of total vector electric field system to be expected from the dynamic part of the transmembrane currents produced by somatic action potential propagation. The static part of the transmembrane electric field was not computed. The electric field system produced by dendritic spiking (synaptic activity) was not computed. The magnetic field system was not computed (although the equations support that computation).

Figure 3 shows still images from two videos of the scalar electric potential field at $12.195 \mathrm{~ms}$ for the Figure 2 planes (a) $\mathrm{Z}=0.0 \mathrm{~mm}$ and (b) $\mathrm{Y}=0.0 \mathrm{~mm}$. The associated videos are provided as supplementary data S6_Test 3 XY.AVI and S7 Test 3 XZ.AVI respectively. These planes pass through the soma and the initial segment of the D151 neuron. Observed fully in three dimensions, it reveals a rotating, pulsing and reversing dipolar electric field that acts like a broad rotating 'lighthouse beam' centered on the region of the initial segment. The field system is weak but despite this, it imposes a spatially large and temporally coherent regularizing influence over a large volume of the space containing the neuron at millimeter scales. In real tissue this means that it would directly interact with itself and thousands of potential neuron neighbors, all of whom produce a similar field system. This produces an extraordinarily complex EM field system throughout the space occupied by tissue, with regularity dependent on spatial and temporal coherence properties within and between the thousands of neurons in any cubic millimeter. 


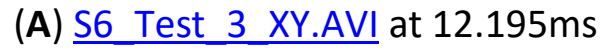

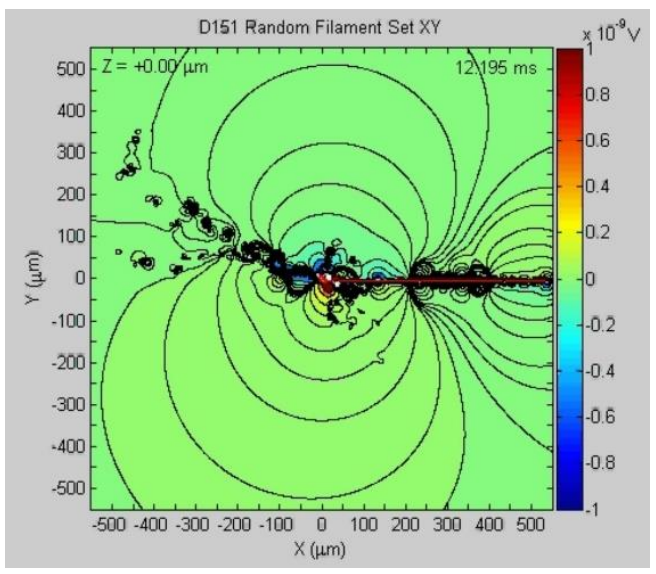

(B) $\underline{\mathrm{S} 7 \text { Test } 3 \mathrm{XZ} . \mathrm{AVI}}$ at $12.195 \mathrm{~ms}$

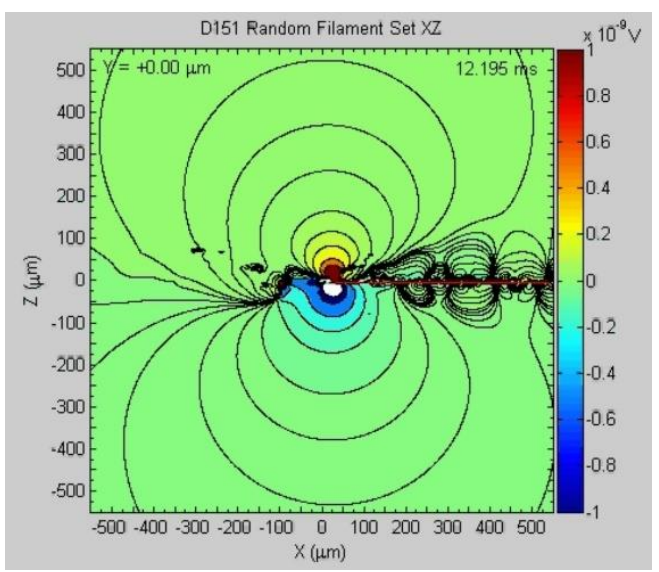

Figure 3. Electric field rendering for the Figure 2 neuron somatic action potential. The computational study produced what are expected to be typical of the general kind of electric field system to be expected from the somatic action potential of excitable cells. It does not show the transmembrane potential. Nor does it show any the field system produced by dendritic action potentials (synapses). The images depict the scalar potential field impressed on space by the electric field produced by the somatic action potential transmembrane currents of the neuron shown in Figure 2 at time $12.195 \mathrm{~ms}$. (A) Shows the $\mathrm{XY}$ plane $(\mathrm{Z}=0)$ and $(\mathbf{B})$ shows the $\mathrm{XZ}$ plane $(\mathrm{Y}=0)$. The full animation of both the $\mathrm{XZ}$ and $\mathrm{XY}$ electric field is provided in supplementary videos. It reveals a pulsing, rotating, growing, and shrinking lighthouse beam effect. The electric field system bathes itself and all its neighbors (at roughly $0.5 \mathrm{~mm}$ scales) in a weak but highly structured, repetitive, and spatially large electric field system that dominates all the molecular field noise produced by the underlying chemistry. In retaining the natural charge and current source geometry, Xchip influences its neighbors naturally and produces the same general kind of transmembrane voltages shown in Figure 2. This makes Xchip form of (ii) empirical science (a replicant).

\subsection{Study 3: The electric field produced by a synapse ion channel plaque}

The third, again novel part of the computational study uses the results of an adaptation of the fieldtheory work to the specialized context of a single synaptic ion channel plaque (Hales et al., 2011). The study was based on a flat post-synaptic plaque of 37 notional ion channel (filament) currents (Figure 4(B)).

This field system is not part of a regenerative, travelling wave (including that triggered by a dendritic action potential). A total post-synaptic 'alpha' current waveform was divided equally over the set of current filaments. The alpha-current waveform is a simple unipolar pulse constructed of two superposed abstract exponential currents.

The resultant electric field is a pulsing dipole with the shape shown in Figure 4(A). The full timecourse of the electric field is shown in the supplementary video Boston 704x576 MPG1 25fps.mpg. This field system is not present in Figures 2 or 3. In reality, thousands of these small dipoles would be located distributed over the entire system of dendrites and the neuron soma. They would each create a localized, superimposed dipole aligned orthogonally to the post-synaptic cleft surface. Combined with the host neuron's SAP field system, any local DAP EM fields and the multitude of EM fields impressed by the thousands of intermeshed nearby neurons, a prodigiously complex, spatially large EM field system is created that can conceivably summate to an intensity with functional significance for all the neurons in the local environment. 
(A) $12.195 \mathrm{~ms}$ snapshot $(\mathrm{Z}=0.0)$

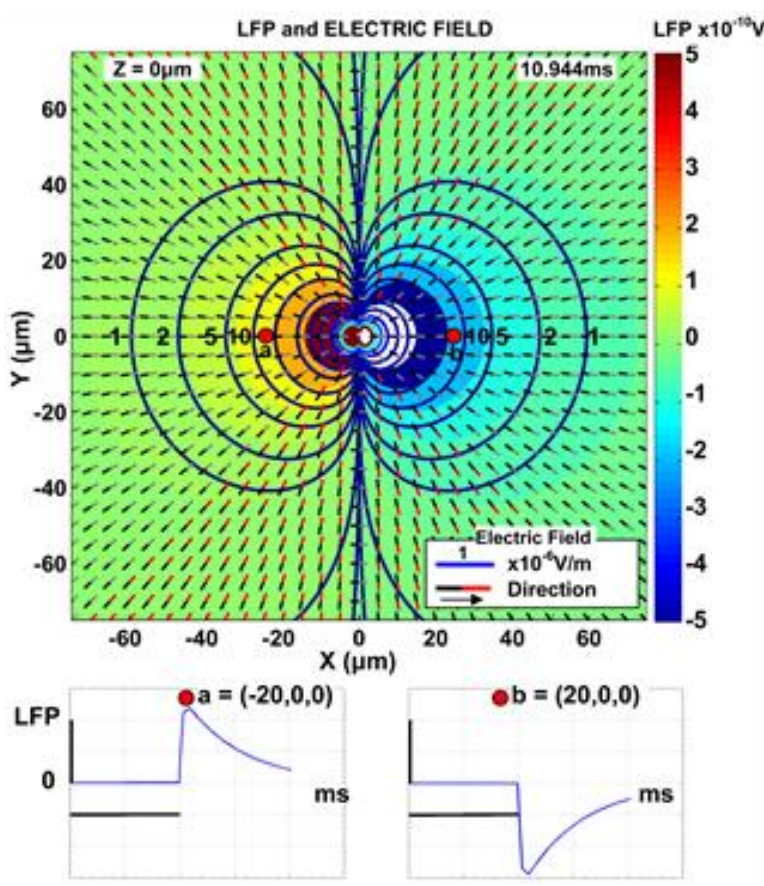

(B) Synaptic plaque

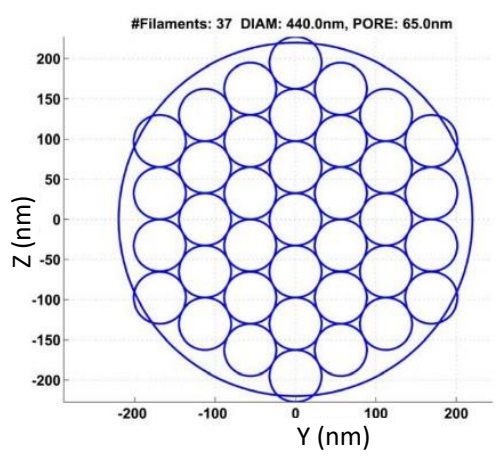

Figure 4. The dipolar electric field of a macular ion channel plaque. (A) LFP and electric field rendering for the (B) post-synaptic filament plaque oriented in the XZ plane. Each filament carries its (equally apportioned) part of an 'alpha current'. The filaments are embedded in an infinite volume conductor. In (A) we have an XY plane snapshot at $\mathrm{t}=10.944 \mathrm{~ms}, \mathrm{Z}=0.0$ of electric field lines and iso-electric field contours. The electric field lines are superimposed on the LFP. Isopotential contours match the dipolar shape of the electric field contours. Electric field vectors are two-colored to give direction. Underneath we have the LFP time course at points a $(-20,0,0)$ and $b(20,0,0)$. Vertical scale bar $2 \times 10^{-10} \mathrm{~V}$. Horizontal scale bar $10 \mathrm{~ms}$. Reproduced with permission from (Hales et al., 2011).

\subsection{Signing off on the Xchip/Neuromorphic empirical/theoretical science issue}

We are now able to technically specify the stark differences between the neuromimetic Xchip and a neuromorphic chip. The difference is that Xchip is constructed to literally generate the electric field activity produced by neurons of the kind revealed in the above study Figures 3 and 4 . Xchip also generates the associated magnetic field system. A neuromorphic chip (Figure 2) does neither. Xchip naturally expresses the transmembrane voltage dynamic using the natural tissue EM field system. Neuromorphic chips are an analogue computer representing the same transmembrane voltage using an EM field system unlike that of natural tissue.

In Xchip the transmembrane currents are accurately traversing a membrane (the dielectric of the capacitor) in the manner of natural membrane. This produces the dynamic electric and magnetic field system of a planar capacitor that occurs when current flows through the (temporarily broken-down) dielectric of the capacitor. This is designed to be physically analogous to cell membrane (a capacitor dielectric with poorly conducting electrolyte plates) penetrated by ion channels. The membranecapacitance discharges through the ion channels, producing a pulsing dipole of the form shown in Figure 4. When thousands of instances of this kind of punctate ion channel transmembrane current/capacitance discharge dipole activity are spread over the surface of a neuron and temporally coordinated in the manner of a propagating somatic action potential, the thousands of Figure 4 temporally coordinated stationary time-varying dipoles summate to produce a total time-varying 
electric field system of the kind depicted in Figure 3. A mature Xchip incorporating many neurons can be expected to produce an EM field system composed of a vector-summated system of whole-neuron EM fields. Systems of closely apposed, intermeshed neurons mutually immerse each other in their EM field systems, potentially mutually altering each other's dynamics. Xchip explores this complexity. The neuromorphic chip does not.

The (Hales, 2011) host study of the natural EM field of neurons revealed it to be highly geometry dependent. If the number of current filaments (ion channels) and their positions on the compartment surface are conserved, but the compartment itself is moved in space (say, rotated), a radical change in the local EM field system can be expected. Examples of this are depicted in the host study. Empirical exploration of these kinds of phenomena is possible with Xchip. A neuromorphic computer cannot do this kind of empirical exploration.

The endogenous EM fields of on-chip devices, to chip designers, are normally 'stray fields' and a source of device malfunction. Part of the traditional chip design process is to eliminate them or minimize their influence. Nature, on the other hand, lacks this overt exogenous intervention and has found a use for the endogenous EM fields. In Xchip we seek, like nature did, to retain the natural expression and functional role of the endogenous EM field system of natural excitable cells. To that end it will require Xchip designers to adopt a new attitude to a functional role for the endogenous EM field systems produced by on-chip devices. This will require new design tools and new kinds of devices.

So far, the discussion has used the neuromorphic GP-computer to contrast its science role with that of the neuromimetic Xchip. We can now generalize the neuromorphic GP-computer to any of the others in Supplementary 1-1. In doing so, the Xchip EM field physics and the GP-computer EM field physics immediately lose all common physics properties (such as voltage). Inside the other GP-computers are abstract (encoded) symbolic representations of properties of brain tissue signaling. However, instead of symbolically representing transmembrane voltage by a measurable analogue voltage, the same voltage is symbolically represented as a digital number stored using circuit element-physics (memory elements) that bears no relation to natural brain physics. The EM field systems of the other GPcomputers have even less relationship to the natural EM field physics of the brain. This reinforces the role of the neuromorphic GP-computer as the optimal benchmark for accurately drawing a distinction between (ii) empirical science (Xchip) and (iii) theoretical science (neuromorphic chip). That distinction easily generalizes to the neuromorphic computer's GP-computer cousins. They too perform Figure 1(e)(iii) theoretical science.

Another way of depicting the deformation of the Figure 1(e) neuroscience as a (ii) $\Leftrightarrow$ (iii) confusion is to imagine that the research communities are notionally placing GP-computers in both the Figure 1(e)(ii) and Figure 1(e)(iii) locations. In doing this, property replication (symbolic modelling) is again being confused with physics replication merely because the same or similar voltages appear (either literally or symbolically) on the chips. With the arrival of Xchip based on accurate physics replication, all GP-computers lose any claim on a (ii) position in Figure 1(e). Their correct location is Figure 1(e)(iii).

A final nuance is in consideration of the inclusion of abstract physics models of the EM fields in Figure 1(e)(iii). If carried out, this would remain an instance of (iii) theoretical science and a valuable route to the testing of functional equivalence of (iii) with (i) and (ii). The examination of equivalence remains unaffected by the expanded modelling. An interesting happenstance exists in the need for the simulation of Xchip physics that occurs when Xchip is being designed. This simulation provides an 
ideal benchmark for (ii) $\Leftrightarrow$ (iii) equivalence testing. The testing still requires a Figure 1(e)(ii) Xchip that literally creates the EM fields, not an abstract model of them. Proof of any (i) $\Leftrightarrow$ (iii) equivalence remains an empirical matter resolved by examination of (ii) $\Leftrightarrow$ (iii) physics/functional equivalence.

It is worth noting that the main results of this article are invariant to the particulars of the origins of EM field expression. Let us say, just for argument's sake, a number of different research groups each had their own theory of how membrane ion channel physics originates the EM field system in tissue. Or perhaps one group includes the action of gap junctions and another hypothesizes that ion channel gating EM fields were important. And so forth. Each group could produce their own neuromimetic chip based on their own assessment of the relative importance of these different EM phenomena. The intent of each group is the same: hardware that captures brain signaling through on-chip hardwarereplication of systems of charge and current density sources that produce the EM field expression of excitable cell tissue. The different groups are all operating in science under (ii), not (iii). The overall implications of this article are therefore agnostic to the particular choices illustrated in the section 3 modeling (EM field origin). We are all still free to argue about the details of the physics. The section 3 modelling could be proved wrong or incomplete in time. This does not matter. The basic claims of this article, about the operation of the science, would not change. The various groups will each support the same basic claims about the kind of science being done, its location inside neuroscience and the implications for AI. The contrast with neuromorphic chips would yield the same conclusion. From this perspective, the particular EM field origin illustrated in section 3 merely serves to exemplify the essence of the fundamental difference in approach that (ii) empirical science presents. If we are to produce an inorganic form of artificial brain tissue, why would we not expect it to replicate brainmimetic EM fields with the same functional relevance? What are you losing if you don't? Nobody knows yet. Nobody can conclusively claim the endogenous EM field expression does not matter. That alone justifies a renewed attention to the operation of (ii) empirical neuroscience and the implications explored herein.

Ultimately, it is the subtle failure in the distinction described above, between nature and abstract models of nature, that is the central technical detail that is revealed to have entrenched itself in the structure of neuroscience for six decades. Its basis is in an understandable confusion of (iii) theoretical science (Figure 1 emulant) with (ii) empirical science (Figure 1 replicant). It explains the Figure 1(e) anomalous form of the science that originated in the 1950s and that appears to have been developed and habituated over the decades. It continued in neuromorphic computers when they originated in the late 1980s. It continues today. All along, the lack of Figure 1(e)(ii) neuroscience was conserved while assuming a (i) $\Leftrightarrow$ (iii) functional equivalence that happens nowhere else in science.

\section{$4 \quad$ Understanding current AI practice}

If we introduce a (ii) Xchip to neuroscience set up in the present-day form of Figure 1(e), we will find ourselves with two different communities of researchers both claiming to be able to potentially create artificial intelligence equivalent to natural intelligence. Superficially, this can be explained as a confusion about what constitutes the 'artificial' part of 'artificial intelligence'. Scientists doing AI under (ii) can validly claim that the (iii) scientists' idea of 'artificial' is dictionary-correct ('made by humans') e.g. (Thorisson, 2020) but is scientifically incorrect because it is not a (ii) scientificallyartificial version of a natural phenomenon. It cannot be. This is because all the (i) natural physics is gone in (iii). However, as revealed above, the structure of the Figure 1(e) science cannot be properly explained as merely a matter of word definitions. The analysis has revealed it to involve subtle matters of physics and the real possibility of a unique, albeit anomalous (i) $\Leftrightarrow$ (iii) equivalence in the neuroscience of excitable cells. 
Let us now assume that the brain is unique and that (i) $\Leftrightarrow$ (iii) equivalence can be expected. If so, then the (i) $\Leftrightarrow$ (iii) equivalence is anomalous in science, but the anomaly is a scientifically valid one. That being the case, there should be a law of nature, a special 'law of physics-independence' (call it $\mathrm{T}_{\mathrm{COMP}}$ ) positing the expectation. There should also be empirical proof of TCOMP, making it a 'law of nature'. That is, $\mathrm{T}_{\mathrm{COMP}}$ must manifest as describing a measurable natural phenomenon critically dependent on it (the measured equivalence of (i) and (iii)). This would then justify the exclusive use of GP-computers and the universal exclusion of attention to (ii) in neuroscience/AI that started in the 1950s and continues today. $\mathrm{T}_{\mathrm{COMP}}$ may well be true. However, note that Xchip, and the (ii) science it supports, needs no extra law of nature to be justified as potentially originating artificial intelligence equivalent to natural intelligence. Nature has already proved that brain signaling physics can originate natural intelligence. Using an inorganic form of the same brain physics under (ii) needs no special justification.

Examination of the literature reveals a few inconsistent and inconclusively hypothesized forms of

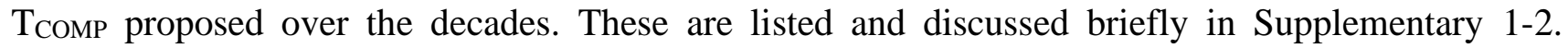
Regardless of the form of $\mathrm{T}_{\mathrm{COMP}}$, it is only when it is empirically proved that AI done with (iii) GPcomputers (lacking all (i) natural physics) can claim access to potential functional equivalence with (i) natural intelligence. As far as can be as ascertained so far, the various existing $\mathrm{T}_{\mathrm{COMP}}$ in the supplementary material are unproved abstract hypotheses that have been assumed true (a 'law of nature') by all researchers. This is another route to understanding the current state of neuroscience and AI. How does the introduction of the (ii) neuromimetic Xchip change the technical conduct of the science?

\section{$5 \quad$ Normalizing the conduct of the science of AI}

Xworld may be forever imaginary, but Xchip is possible. We can now do a detailed comparison of how adding Xchip as a (ii) empirical neuroscience option normalizes the science of AI, and how this addition relates to a proof of the 'law of physics-independence' (TCOMP). Because of the introduction of Xchip there are two forms of the science of AI:

\section{1 (1) Tcomp proof by exhaustion (XChip not available).}

The method targets AI using (iii) GP-computers, thereby proving $\mathrm{T}_{\text {COMP }}$ by abstract modelling of natural brain tissue at a physical or functional level that is then compared with (i) natural intelligence. As noted in the introduction, the abstract models are not limited to neuroscience e.g. (Hassabis et al., 2017). The specific form of TCOMP is irrelevant. Whatever it is, it is assumed true. Methodologically this TCOMP proof process is what we have been doing in AI since its inception: testing an hypothesis (assumed true) that an artificial form of natural intelligence functionally equivalent to natural fauna can be achieved with GP-computers and abstract models under Figure 1(e)(iii). So far it has failed. The failure is interesting and nuanced. It is detailed in Supplementary 1-3. At this point in time, the empirical work has delivered 65 years of successful and powerful automation technology ('narrow-AI' (Goertzel, 2015)) while at the same time delivering ambiguous counterevidence in respect of $\mathrm{T}_{\mathrm{COMP}}$ and (i) $\Leftrightarrow$ (iii) equivalence. For reasons listed in Supplementary 1-3, conducting the science of AI in this way proceeds while delivering impoverished guidance as to the necessary kind of (or repairs to) abstract models and the necessary quantity of GP-computing resources needed to achieve (i) $\Leftrightarrow$ (iii) equivalence. What GP-computer resourcing will it take to prove $T_{\text {COMP? }}$ ? Whatever that is, the existing 'proof by exhaustion' technique is methodologically committed to delivering it to find out. 


\section{2 (2) Tcomp proof by examination of the null hypothesis (XChip available).}

Here the potential equivalence of (iii) model and (i) nature is explored progressively and discovered. The method's 'null hypothesis' empirical approach involves the inclusion of the Figure 1(e)(ii) option that does not use GP-computers. Unlike (1), the method does not assume $\mathrm{T}_{\mathrm{COMP}}$ is true, i.e. that there is no (i) physics essential to natural function. The method uses all three normal science contexts, (i)(iii). These are Xchip ((ii) empirical science) in comparison with both GP-computers ((iii) theoretical science) and the natural brain ((i) empirical science). When option (ii) is included using Xchip, the testing explores equivalence at the physics level, not the functional level. Function can be inferred as lost or altered when physics equivalence is lost (this occurs when physics model predictions and observation part company). The ambiguity in failure in (1) is avoided because failure of a model is delivered with empirically tractable (explorable) physics advice as to why the failure occurred. We can then relate the loss to any impact on function. This ((ii) empirical science) process delivers us the necessary corrections to a failed model ((iii) theoretical science). Access to this extra information is absent in (1). In this way, the context of the potential truth of $\mathrm{T}_{\mathrm{COMP}}$ is discovered by empirical exploration, not presupposed. Paradoxically, the null-hypothesis method also reveals that in order to conclusively prove that GP-computers may be all we need to create AI (albeit anomalously), AI done without using GP-computers is an essential part of the empirical proof. This method also delivers, early in comparison to (1), information about the GP-computer resources needed in (iii) modelling (GPcomputing) the physics underlying (i) natural function. This is the form of the neuroscience of AI normalized to include (ii).

\subsection{Discussion: (1) and (2) over the decades}

We now have 65 years of option (1). Option (2), as far as can be ascertained to date, has been missing for the same duration. Compounding the situation is that the sciences of neuroscience and AI are unknowingly in the (1) state. That is, the literature also reveals no detectable awareness of (2) as a possibility. In one sense this lack of awareness is to be expected. The technology and knowledge levels within neuroscience at the time of the birth of AI made (2) impossible. However, the science of AI could have proceeded as per (1) while being aware that (2) was potentially possible. For reasons not known, this did not happen. This article appears to be the first time (2) has been thoroughly explored as a possibility. The science of AI simply appeared in the 1950s in the (1) form, exclusively using GPcomputers within computer science, itself a nascent discipline. Emerging alongside it was the long and controversial literature trail discussed in the introduction. This literature trail could be interpreted as an expression of some kind of general awareness of the lack of (2) within the science. From that perspective this article serves to bring the lack of (2), and its relationship with the (i) brain/(iii) GPcomputer equivalence argument, into focus in a helpful, revealing way. Meanwhile, in the absence of (2), the (1) approach still regularly sees the science of AI engaged in (again inconclusively, so far) justifications of how its activities are connected to the successful attainment of its stated aims. These aims have recently been restated and re-examined (Monett et al., 2020). GP-computers and (1) continue to define the conduct of the science.

With the neuromimetic Xchip, the (2) form of the conduct of the science of AI is possible in principle because the underlying neuroscience has been normalized through the populating of Figure 1(e)(ii). In 2020 we have the neuroscience knowledge and the chip foundry technology needed to build the progenitors of Xchip at scale with brain tissue. The now overprepared alternative, (2), can no longer tacitly/habitually lack due attention by neuroscience or AI. To continue with the Figure1(e) form of the science, we must construct an explicit argument that justifies continued confinement to (1) even 
though (2) is possible. The days of a methodologically imposed 'benefit of the doubt' (the assumption that $\mathrm{T}_{\text {COMP }}$ is true), while underperforming in the manner detailed in Supplementary 1-3, are over.

Note that none of the above discussion is intended to imply that GP-computers cannot reach equivalence with natural brain function under circumstances not yet understood. That potentiality is not the issue here and is not contested. The issue here is how neuroscience and the science of AI must be configured to empirically determine any potential equivalence and the context in which it may happen. With method (1) the equivalence is presupposed. With method (2), the equivalence of GPcomputers and brains can be empirically settled by a fully formed, normal neuroscience.

\subsection{Embodiment, embeddedness, Xchip and the final form of the science}

A final nuance is that Xchip can only be developed and proved as a robotics project. Inorganic 'physics of embodiment' (a robot body with a peripheral nervous system) is as mandatory as the claimed essential physics within its Xchip brain (central nervous system). Embeddedness within a test environment is also naturally mandated. The natural brain exists embodied and that body is embedded in an environment. Xchip inherits the same natural situation. Supplementary 2 depicts the developmental embodied/embedded AI test situation containing both an Xchip-based robot and a GPcomputer-based robot. These two test subjects are compared/contrasted in a fully normalized neuroscience that includes the Figure 1(e)(ii) empirical component previously missing from the science. Further details will emerge in the future prototyping work.

\section{Summary}

It has been shown that, with modern chip foundry technology and neuroscience knowledge, neuroscience can in principle create a neuromimetic chip (Xchip) as a form of inorganic artificial brain tissue based on a 'physics-replication' approach. It is comprised entirely of an inorganic form of excitable cell signaling electromagnetism (EM) that replicates the fundamental physics that originates the endogenous EM field system of natural excitable cell tissue. This EM field system facilitates the natural expression of all the signaling system types found in the brain: somatic/axonal and dendritic action potentials (membrane-longitudinal, dromic and anti-dromic), the naturally associated and simultaneously produced ephaptic coupling (membrane-transverse, both electric and magnetic fields) and EM field-mediated synaptic connections.

When applied to machine intelligence, Xchip naturally creates 'neuroscience-originated' AI that lacks the usage of general-purpose computers, abstract models, and software. The hallmark of the approach is that a fully developed human-level Xchip would emanate an EM field system that could in-principle be measured with the same EEG and MEG equipment used on humans.

Xchip's EM field system has the same functional significance as the endogenous EM field system of natural excitable cell tissue, which is known to originate in the membrane-embedded ion channel activity of single neurons (See Supplementary 1 references). Fundamentally, the physical change at the on-chip device level is rather trivial: it is a simple structural change to the planar capacitor used to replicate neuron membrane as detailed in section 2.3 and Supplementary 1 . This article only provides a plausible conceptual basis for Xchip. Further Xchip technical design detail must await a significant empirical exploration. Even if this detail were available now, it would not change the basic thrust of the arguments herein, which are about an anomalously configured science for which any Xchipdirected activity is corrective. Regardless of Xchip design detail, the cultural implications of the change, in terms of the naturalization of the EM field expression, are profound. No known existing chip deliberately expresses a membrane-realistic EM field system. The Xchip concept is based on it. 
The fundamental membrane physics was presented in section 3. Its mathematical basis (Maxwell's equations) was developed in the original study referenced herein. While the physics basis is relatively straightforward, it is the deeper surrounding cultural matters that have resulted in the need for this article.

Replicating brain electromagnetism constitutes a faithful replication of natural brain tissue function. This was called a 'scientifically-artificial' version of natural brain tissue in the same way a dialysis machine is a 'scientifically-artificial' version of natural kidney tissue. The contemporary lack of such an approach, in a general science sense, was identified as unique, unprecedented, and anomalous in science. It is methodologically equivalent to expecting to fly (see Figure 1(b)) while never actually using any flight physics and assuming, without any principled reason explored by experimentation with flight physics, that flight can be achieved by disposing of flight physics through completely replacing it with the physics of a general-purpose computer, a state of 'physics-independence' not found in any other natural physics context. This sounds like a harsh depiction of the science. It is merely a realistic description of the Figure 1(e) situation we all currently inhabit. The brain may well be the first (and perhaps only) instance of a natural phenomenon for which this special 'physics-independence' property may apply (a 'law of nature' called $\mathrm{T}_{\text {COMP }}$ in the above analysis). That being the case, existing neuroscience was demonstrated to be lacking the normal methodological structure needed to empirically prove it. Empirical proof requires the natural brain physics to be retained for the purposes so that its controlled lack can be assessed. It is the controllable retention of the natural physics that Xchip delivers. The origin of the lack of the physics-replication approach was traced as far back as the time of the birth of general-purpose computers and the inception of AI in the 1950s. The reader is encouraged to contact the author if they have knowledge of a single instance, in the science literature, of any signs of even a proposal for the physics-replication empirical technique outlined here. Such a find would be significant and greatly appreciated.

Xchip's introduction of a normal empirical option that is anomalously absent, was couched as a normalization of neuroscience and, by extension, a normalization of AI. In development projects tackling this kind of neuromimetic chip, empirical results, and their position within and contribution to neuroscience/AI will necessarily require an understanding of the issues raised here. Electrical engineers tasked with prototyping Xchip will need to be apprised of the implications. Neuroscience, electrical engineering and computer science must be thoroughly conversant with the full science framework formalized here and integrate their respective knowledge bases directly and accurately if the Xchip concept is to reach its potential and prove it.

\section{$7 \quad$ Conflict of Interest}

The authors declare that the research was conducted in the absence of any commercial or financial relationships that could be construed as a potential conflict of interest.

\section{Author Contributions}

The author is the sole originator of this article and the supporting material.

\section{$9 \quad$ Funding}

This work has been carried out with support of 'Anatomy \& Neuroscience Pilot Grant' 39-4020-GEN awarded to the author by the University of Melbourne's Department of Anatomy and Neuroscience.

\section{Acknowledgments}


Thanks go to Andrea Varsavsky, Natesh Ganesh and Dagmar Monett for valuable commentary on earlier incarnations of this article. It is Peter Kitchener's persistence, both as a 'bulldog' and 'devil's advocate', that have been pivotal in finding the underlying structure of the argument and helping me to get it to speak clearly to its target audiences.

\section{References}

Brooks, R., Hassabis, D., Bray, D., and Shashua, A. (2012). Is the brain a good model for machine intelligence? Nature 482, 462.

Dreyfus, H.L. (1972). What computers can't do: A critique of artificial intelligence. Harper \& Row.

Fjelland, R. (2020). Why general artificial intelligence will not be realized. Humanities and Social Sciences Communications 7, 1-9.

Goertzel, B. (2015). Artificial General Intelligence. Scholarpedia 10, 31847.

Hales, C.G. (2011). Doctoral Thesis: The Electric Field System of an Excitable Cell. Dept. of Electrical and Electronic Engineering, University of Melbourne.

Hales, C.G. (2014). The Revolutions of Scientific Structure. Singapore: World Scientific.

Hales, C.G., Grayden, D.B., and Quiney, H.M. (2011). "The Electric Field System of a Macular Ion Channel Plaque", in: 33rd Annual International Conference of the IEEE Engineering in Medicine and Biology Society (EMBC 2011). (Boston, Mass. USA).

Hales, C.G., and Pockett, S. (2014). The relationship between local field potentials (LFPs) and the electromagnetic fields that give rise to them. Frontiers in Systems Neuroscience 8, 233.

Hassabis, D., Kumaran, D., Summerfield, C., and Botvinick, M. (2017). Neuroscience-inspired artificial intelligence. Neuron 95, 245-258.

Hines, M.L., and Carnevale, N.T. (1997). The NEURON simulation environment. Neural Computation 9, 1179-1209.

Hines, M.L., and Carnevale, N.T. (2001). NEURON: A tool for neuroscientists. The Neuroscientist 7, 123-135.

Hines, M.L., and Carnevale, N.T. (2003). "The NEURON simulation environment," in The handbook of brain theory and neural networks, ed. M.A. Arbib. 2nd ed (Cambridge, Mass.: MIT Press), 769-773.

Mccarthy, J., Minsky, M., Rochester, N., and Shannon, C.E. (1955). A PROPOSAL FOR THE DARTMOUTH SUMMER RESEARCH PROJECT ON ARTIFICIAL INTELLIGENCE [Online]. Available: http://www-formal.stanford.edu/jmc/history/dartmouth/dartmouth.html [Accessed].

Milkowski, M. (2017). Why think that the brain is not a computer? APA Newsletter 16.

Monett, D., Lewis, C., and Thorisson, K.R. (eds.). (2020). Journal of Artificial General Intelligence special issue of commentaries on "On Defining Artificial Intelligence” by Pei Wang.

Moor, J. (2006). The Dartmouth College Artificial Intelligence Conference: The next fifty years. $A i$ Magazine 27, 87-91.

Rosenblueth, A., and Wiener, N. (1945). The role of models in science. Philosophy of science 12, 316-321.

Searle, J.R. (1980). Minds, Brains and Programs. Behavioral and Brain Sciences 3, 417-425. 
Thorisson, K.R. (2020). "Discretionarily Constrained Adaptation Under Insufficient Knowledge \& Resources," in Journal of Artificial General Intelligence special issue of commentaries on "On Defining Artificial Intelligence” by Pei Wang (Vol 11, Issue 2), eds. D. Monett, C.W.P. Lewis \& K.R. Thorisson. Sciendo), 7-12.

Turing, A. (1964). "Computing machinery and intelligence," in Minds and Machines, ed. A. Anderson. (Englewood Cliffs, NJ: Prentice Hall), 4-30.

Turing, A.M. (1950). Computing machinery and intelligence. Mind 59, 433-460. 